\title{
Fast calculation of direct fire trajectories taking the earth's rotation into account
}

\author{
W. Roetzel ${ }^{1}$, W. Czarnetzki ${ }^{2}$ \& T. Maier ${ }^{2}$ \\ ${ }^{1}$ Helmut-Schmidt-Universität / Universität der Bundeswehr Hamburg, \\ Germany \\ ${ }^{2}$ Hochschule Esslingen, Germany
}

\begin{abstract}
An analytical approach is developed for the subsequent consideration of the Coriolis effect. The target is regarded as a moving target in the star-fixed coordinate system. The stationary atmosphere creates a nonuniform cross-wind which reduces the normal moving target deflection of the projectile. The approach is implemented in a previously developed analytical fast calculation method and tested against numerical calculations with good results.

Keyword: Coriolis effect, analytical solution, power law, drag coefficient, Mach number.
\end{abstract}

\section{Introduction}

Several analytical solutions of the point mass equation of motion have been developed for the fast calculation of direct fire trajectories [1-4], which are based on the power law

$$
c_{D}=C \cdot(M a)^{-m}=C \cdot\left(\frac{a}{v}\right)^{m}=C \cdot\left(\frac{a^{*}}{v}\right)^{m} \cdot\left(\frac{T}{T^{*}}\right)^{m / 2}
$$

for the drag coefficient variation with Mach number. McCoy [1] published flat fire solutions for $m=0,1 / 2,1$. The later solutions [2-4] allow for arbitrary values of $m$ and any angle of sight $\beta[3,4]$. Wind can be considered using a coordinate transformation $[2,4]$. For uphill and downhill firing the change of pressure and temperature along the trajectory should be considered. Usually mean values of 
pressure and temperature are applied [3]. A more sophisticated way [4] is the correction of the Mach number exponent, which takes not only the changing pressure and temperature but also the curvature of the trajectory approximately into consideration.

The above mentioned fast calculation methods do not allow for the earth's rotation. In the present paper a simple analytical method is described [5] with which the Coriolis effect can subsequently be considered. The same coordinate system and nomenclature is used as in the foregoing paper [4].

\section{Numerical method}

For the precise calculation of the trajectory the complete equation of motion has to be integrated numerically. With the previously $[2,4]$ defined ballistic coefficient

$$
D(p, T)=C \cdot \frac{\pi d^{2}}{8 M} \rho a^{m}=C \cdot \frac{\pi d^{2}}{8 M} \rho^{*}\left(a^{*}\right)^{m} \cdot \frac{p}{p^{*}} \cdot\left(\frac{T^{*}}{T}\right)^{1-m / 2}
$$

the equation of motion with wind velocity $\vec{w}$ can be written as [1]

$$
\dot{\overrightarrow{\mathrm{v}}}=\vec{g}-D \cdot(|\vec{v}-\vec{w}|)^{1-m} \cdot(\vec{v}-\vec{w})-\vec{b}
$$

with the Coriolis acceleration

$$
\overrightarrow{\mathrm{b}}=-2 \vec{\omega} \times \vec{v}
$$

containing the earth's vector $\vec{\omega}$ of rotation

$$
\vec{\omega}=\omega\left\{\begin{array}{c}
\cos \psi \cos \gamma \\
\sin \psi \\
-\cos \psi \sin \gamma
\end{array}\right\} .
$$

In eqn (5) the angle $\psi$ is the latitude and the angle $\gamma$ the azimuth of fire (x-axis), measured clockwise from north. Assuming the standard temperature drop with altitude $\Delta T / \Delta y=-0.0065 \mathrm{k} / \mathrm{m}$ [6] and regarding the atmosphere as perfect dry air yields the ballistic coefficient $D$ as function of height $y$

$$
D=D_{0} \cdot\left(1-\frac{0.0065}{T_{0}} \cdot y\right)^{4.25593+m / 2},
$$

where index " 0 " indicates the firing site and origin of the earth-fixes coordinate system. 
Starting at the origin with $\tau=0$ and $\vec{L}=\{x, y, z\}=0$, the local projectile velocity $\vec{v}$ can stepwise be calculated as function of time. Integrating simultaneously over the time yields $\vec{L}$ as function of time. This numerical calculation is later carried out in order to test the new approach which is derived and described in the following.

\section{Analytical approach}

The equation of motion without the Coriolis acceleration is valid in a star-fixed not rotating system which moves with uniform velocity. Therefore the coordinate system in which the Coriolis-free solutions [1-4] are valid is considered to move on uniformly with the fixed circumferential (not rotating) velocity $\vec{u}_{0}$ of the firing site (origin of coordinate system) at the instant of firing $\tau=0$. For $\tau=0$ this star-fixed coordinate system coincides with the earth-fixed system, for $\tau>0$ they separate from each other.

\subsection{The moving centre of gravity}

In this star-fixed system a flying projectile experiences an additional gravitational acceleration $\Delta \vec{g}$ in the horizontal direction, as the centre of gravity does not remain exactly perpendicular below the projectile. With the simplifying assumption of constant mean velocity components $v_{x}$ and $v_{z}$ and the radius $R=$ $6.37 \cdot 10^{6} \mathrm{~m}$ of the globe, the additional time dependent acceleration can be expressed as

$$
\Delta \vec{g}=-\frac{g \cdot \tau}{R}\left\{\begin{array}{c}
u_{x}+\bar{v}_{x} \\
0 \\
u_{z}+\bar{v}_{z}
\end{array}\right\}=-\frac{g \cdot \tau}{R}\left\{\begin{array}{c}
-\omega_{z} \cdot R+x_{1} / \tau \\
0 \\
\omega_{x} \cdot R+z_{1} / \tau
\end{array}\right\} .
$$

Substituting $\omega_{\mathrm{x}}$ and $\omega_{\mathrm{z}}$ according to eqn (5) and integrating twice over the time of flight yields the displacement of the projectile

$$
\Delta \vec{L}_{1 g}=-\frac{g}{6} \cdot \tau_{1}^{3}\left\{\begin{array}{c}
\omega \cos \psi \sin \gamma+x_{1} / R \tau_{1} \\
0 \\
\omega \cos \psi \cos \gamma+z_{1} / R \tau_{1}
\end{array}\right\} .
$$

The vertical components $\Delta g_{y}=0$ and $\Delta L_{1 g y}=0$ since $\cos \omega \tau_{1} \approx 1$. The derivation of eqn (8) is correct under vacuum conditions. The effect of eqn (8) is very week in normal cases and a sufficiently accurate approach.

\subsection{The moving target}

The main effect of the earth's rotation is the fact that in the uniformly moving star-fixed system the earth-fixed target appears as a moving target. We look first at the poles of the globe. There the circumferential velocity is zero, but the earth- 
fixed coordinate system rotates relatively to the considered star-fixed system. At the north pole any target will travel from west to east, i.e. from right to left. A negative angle of lead $\alpha<0$ would be required to hit the moving target. The shift $\Delta \vec{L}_{1 \omega}$ of the target during the time of flight $\tau_{1}$ can be expressed as

$$
\Delta \vec{L}_{1 \omega}=\tau_{1}\left[\vec{\omega} \times\left(\vec{L}_{1}+\Delta \vec{L}_{1 g}\right)\right]=\tau_{1}\left[\left(\vec{\omega} \times \vec{L}_{1}\right)+(\underbrace{\vec{\omega} \times \Delta \vec{L}_{1 g}}_{\approx 0})\right]
$$

which leads with eqn (5) to

$$
\Delta \vec{L}_{1 \omega}=\omega \cdot \tau_{1}\left\{\begin{array}{c}
z_{1} \sin \psi+y_{1} \cos \psi \sin \gamma \\
-x_{1} \cos \psi \sin \gamma-z_{1} \cos \psi \cos \gamma \\
y_{1} \cos \psi \cos \gamma-x_{1} \sin \psi
\end{array}\right\} .
$$

At other latitudes $\psi \neq \pm 90^{\circ}$ an additional target movement takes place in the star-fixed system which is caused by the growing distance between the origins of both coordinate systems. However, a detailed analysis shows that this additional shift can be omitted. Its effect is compensated by the fact that in the usual standard free-fall acceleration $\mathrm{g}=9.80665 \mathrm{~m} / \mathrm{s}^{2}$ (at sea level) the centrifugal acceleration due to the earth's rotation is included (subtracted from the mass attraction force). Thus, eqn (10) is valid for all values $-90^{\circ}<\psi<+90^{\circ}$.

The same shift (10) with negative sign is found by the twofold integration of the Coriolis acceleration eqns $(4,5)$ using a constant mean projectile velocity $\vec{v}=\vec{L}_{1} / \tau_{1}$. The negative sign shows that in the earth-fixed system the projectile is apparently accelerated and displaced in the opposite direction as the target is in the star-fixed system. One could suppose that the final Coriolis deflection of the projectile in the earth-fixed system could be expressed as

$$
\Delta \vec{L}_{1}=\Delta \vec{L}_{1 g}-\Delta \vec{L}_{1 \omega} .
$$

However, this is only a rough approximation under normal shooting conditions which will be discussed later in this paper. Eq (11) holds true in a vacuum. The contribution $\Delta \vec{L}_{1 g}$ is normally relatively small. In the special case of the downward free-fall in a vacuum at the equator $\left(\psi=0, \vec{L}_{1}=\left\{0,-L_{1}, 0\right\}\right) \Delta \vec{L}_{1 g}$ is decisive. For $\gamma=0$ and with the time of flight $\tau_{1}=\left(2 L_{1} / g\right)^{1 / 2}$ eqn (11) yields the correct [7, p.214] east drift

$$
\Delta L_{1 z}=\omega \cdot L_{1} \cdot\left(\frac{2 L_{1}}{g}\right)^{1 / 2} \cdot\left(-\frac{1}{3}+1\right)=\omega \cdot L_{1} \cdot\left(\frac{2 L_{1}}{g}\right)^{1 / 2} \cdot \frac{2}{3} .
$$

The term $-1 / 3$ is due to $\Delta \vec{L}_{1 g}$. Neglecting it would cause an overestimation of $50 \%$. 


\subsection{The Coriolis wind}

The moving target problem under consideration differs from the usual case in so far as the air follows the moving target. If no wind is present, the stationary air in the earth-fixed system rotates in the star-fixed system together with the target. The moving target gets tailwind of equal velocity. The wind is not uniformly distributed but the cross-wind velocity $\vec{w}_{c}$ is zero at the firing site and grows linearly with the distance to its maximum value $\vec{w}_{1}$ at the target

$$
\vec{w}_{\mathrm{c}}=\frac{x}{x_{1}} \cdot \overrightarrow{\mathrm{w}}_{1}=\frac{x}{x_{1}} \cdot \frac{-\Delta \vec{L}_{1}}{\tau_{1}} .
$$

This Coriolis wind pushes the projectile towards the moving target and reduces the deflection according to eqn (11). Since the consideration of uniform wind is no problem, a suitable mean uniform Coriolis wind velocity $\vec{w}_{c m}$ is defined and derived which produces the same effect as the actual linearly growing Coriolis wind does:

$$
\overrightarrow{\mathrm{w}}_{\mathrm{cm}}=f \cdot \overrightarrow{\mathrm{w}}_{1}=-f \cdot \frac{\Delta \vec{L}_{1}}{\tau_{1}}==f \cdot \frac{\Delta \vec{L}_{1 \omega}-\Delta \vec{L}_{1 g}}{\tau_{1}} .
$$

A horizontal flat shot in the $\mathrm{x}$-direction $\left(v_{x}=v\right)$ towards a target at distance $x_{1}$ with cross-wind of velocity $w_{z}=w_{c}$ is considered. Gravity is neglected. For $w_{Z}<<v$

$$
\dot{\mathrm{v}}_{\mathrm{z}}=-\frac{\dot{v}}{v}\left(w_{1} \cdot \frac{x}{x_{1}}-v_{z}\right)
$$

From eqns (1-3) one can derive [2]

$$
\frac{x}{x_{1}}=\frac{v_{0}^{m}}{D m x_{1}}\left\{1-\left[1+D(1-m) v_{0}^{1-m} \cdot \tau\right]^{\frac{m}{1-m}}\right\}
$$

and

$$
\frac{\dot{v}}{v}=\frac{-D v_{0}^{1-m}}{1+D(1-m) v_{0}^{1-m} \cdot \tau} .
$$

Substituting eqns (16) and (17) into eqn (15) yields a first order linear inhomogeneous differential equation for $v_{z}(\tau)$. Solving first the homogeneous differential equation and applying the variation of the constant yields with the boundary condition $\tau=0: v_{z}=0$ the projectile velocity component $v_{z}$ as function of time. Integrating $v_{Z}$ from $\tau=0$ to $\tau_{1}$ gives the deflection $z_{1}$ of the projectile at the distance $x_{1}$. The same deflection $z_{1}$ has to be produced by the 
uniform wind of velocity $w_{c m}$, defined with eqn (14). This deflection can be found by replacing in eqn (15) the variable velocity $w_{1} \cdot x / x_{1}$ by the constant velocity $f \cdot w_{1}$. The solution leads to the known formula of Didion [1]

$$
\mathrm{z}_{1}=\frac{f \cdot w_{1}}{v_{0}}\left(v_{0} \cdot \tau_{1}-x_{1}\right)
$$

Equating both deflections $z_{1}$ and solving for $f$ yields finally the implicit formulas for the determination of the correction factor $f$ :

$$
\begin{gathered}
f(V, m)=\frac{V}{V-1} \cdot\left(\frac{V+\frac{m}{1-m}}{m \cdot \eta}+\frac{V-V[1+(1-m) \eta]^{\frac{1-2 m}{1-m}}}{m(1-m)(1-2 m) \cdot \eta^{2}}\right), \\
V=\frac{m \cdot \eta}{1-[1+(1-m) \eta]^{\frac{m}{m-1}}} .
\end{gathered}
$$

For given values of $m$ and guessed values of $\eta$ the dimensionless number $V$ and the factor $f$ can be calculated. Iteratively one can determine $f$ as function of $m$ and $V$. The number $V$ is defined as

$$
\mathrm{V}=\frac{v_{x 0} \cdot \tau_{1}}{x_{1}}=\frac{\tau_{1}}{\tau_{1, p=0}} \geq 1 .
$$

It is the ratio of the horizontal components of the muzzle velocity and the mean velocity. It can be determined once the trajectory has been calculated numerically or analytically (neglecting the Coriolis effect). In a vacuum $V \rightarrow 1$. The right-hand definition as the ratio of the times of flight under normal and vacuum conditions is more appropriate when both $v_{x 0}$ and $x_{1}$ turn to zero. (e.g. free-fall in a vacuum).

In the special case $m=1 / 2$ the variable $\eta$ in eqns $(19,20)$ can be eliminated yielding the explicit formula

$$
f_{m=1 / 2}=\left(\frac{V}{V-1}\right)^{2} \cdot\left(\frac{V+1}{V}-\frac{2 \cdot \ln V}{V-1}\right) .
$$

In the special cases $m=0$ and $m=1$ the eqns $(19,20)$ cannot be applied directly and the following implicit equations have to be used

$$
\begin{gathered}
f_{m=0}=\frac{V}{V-1} \cdot\left(1-\frac{2(V-1)}{\lambda}\right), \quad V=\frac{\lambda}{\ln (1+\lambda)}, \\
f_{m=1}=\frac{V}{V-1} \cdot\left(\frac{2(V-1)}{\mu}-1\right), \quad V=\frac{\mu}{1-e^{-\mu}} .
\end{gathered}
$$


For $\mathrm{m} \neq 1 / 2$ the determination of $f$ is inconvenient and time consuming. Therefore the following empirical explicit formula has been developed:

$$
\frac{1}{f}=1+\frac{2}{V^{E}}, E=0.48(1+m)+0.08 m^{2} .
$$

For the limiting value $V \rightarrow 1$ the exponent $E=(1+m) / 2$ is more precise. Equation (25) is sufficiently accurate for all direct fire applications.

Once the correction factor $f$ is found, the Coriolis deflection of a projectile can subsequently be calculated as follows: First $\Delta \vec{L}_{1}$ is calculated from eqs ( 8 , $10,11)$. This vector represents the deflection of the projectile from the target $\vec{L}_{1}$ neglecting the Coriolis wind. The mean Coriolis wind velocity $\vec{w}_{c m}$ is calculated using eqn (14) with $f$ from eqn (25). If a real uniform wind is present, the Coriolis wind velocity has simply to be added to the real wind velocity. The sum of both winds can be taken into account by a coordinate transformation $[2,4]$ or other known methods.

If no real wind is present the application of Didion's formula is recommended. Combining the shift $\Delta \vec{L}_{1}$ and the Coriolis wind effect according to Didion leads to the following formula for the Coriolis drift of the projectile

$$
\Delta \vec{L}_{1 c}=\Delta \vec{L}_{1} \cdot \frac{2+V^{E-1}}{2+V^{E}} .
$$

A horizontal flat shot has been assumed in the above derivations. For uphill and downhill fire the exponent $\mathrm{m}$ in eqs. (19-26) has to be corrected for changing air pressure and temperature along the trajectory [4]. The exponent $\mathrm{m}$ has to be replaced by $m^{*}=m-n$ with $n$ according to [4, eq. (26)]:

$$
n=\frac{v_{0}^{m} \sin \beta}{D_{0}}\left[\frac{g(1-m)}{v_{0}^{2}}+\frac{(0.0065)(4.25593+m / 2)}{T_{0}}\right] .
$$

\section{Test of the analytical approach against numerical calculations}

The analytical Coriolis approach has been implemented into a fast calculation programme, based on the previously developed explicit Coriolis-free analytical solution [4, chapter 3.2 and 4]. The programme is constructed in such a way that for a given target $\left(x_{1}, y_{1}\right)$ or $\left(L_{1}, \beta\right)$ and wind velocity $\left(w_{x}, w_{y}, w_{z}\right)$ the angle of lead and the gun elevation angle above line of sight is calculated iteratively. The extended programme has been tested against numerical calculations described in chapter 2 of this paper. Four typical examples are presented in the following. 


\subsection{Example 1}

The 185 Grain match projectile Lapua Scenar $.30 \mathrm{~GB} 423, d=7.8232 \mathrm{~mm}, M=$ $11.988 \mathrm{~g}$ is considered with $m=0.4855$ and $C=0.4286$ (eq. (1)). The projectile is fired with $v_{0}=930 \mathrm{~m} / \mathrm{s}$ at sea level, $p_{0}=1013.25 \mathrm{mbar}$ and $t_{0}=15^{\circ} \mathrm{C}$. The angular velocity of the earth is taken as $\omega=7.292 \cdot 10^{-5} \mathrm{rad} / \mathrm{s}$ [1]. The muzzle Mach number $M a_{0}=2.73$. It reduces to $M a_{1}=1.25$ (in all examples) after the fixed time of flight $\tau_{1}=1.6 \mathrm{~s}$. Table 1 shows calculated Coriolis deflections for flat fire from the north pole and the equator. The same results are obtained (with millimeter accuracy) from the analytical and the numerical method. The analytical deflections are calculated using eq. (26) yielding the same values as the fast calculation programme.

\subsection{Example 2}

The same projectile and data are used as in example 1 with the exception of the gun elevation angle $\varphi_{0}=46^{\circ}$ and the latitude $\psi=45^{\circ}$. Table 2 shows the results. The values in brackets are analytically calculated deflections which deviate from the corresponding numerical values. The largest deviation is $1 \mathrm{~mm}$ in height at the distance (bee-line) of about $1 \mathrm{~km}$.

\subsection{Example 3}

A projectile of identical shape (equal values of $\mathrm{m}$ and $\mathrm{C}$ ) and mass density is considered, which has the threefold diameter $\mathrm{d}=23.47 \mathrm{~mm}$. The mass is enlarged by the factor 27, yielding $M=323.68 \mathrm{~g}$. From the analytical solution [4] one can see that with the same muzzle velocity and threefold time of flight $\tau_{1}=$ $4,8 \mathrm{~s}$ the end velocity will remain the same at the threefold distance. So $M a=$ 2.73 to 1.25 . One can also predict that for flat fire the elevation angle above line of sight will increase by the factor 3 . Table 3 shows the flat fire results for $\varphi_{0}=$ $2^{\circ}, \tau_{1}=4.8 \mathrm{~s}$ and $L_{1}=3019.26 \mathrm{~m}$. The largest deviations are $3 \mathrm{~mm}$. Concerning the superelevation angles: For example 1 the Coriolis-free superelevation angle $\varepsilon_{1}=9.7866$ mils and for example $3 \varepsilon_{3}=29.3598$ mils $=$ $3 \cdot \varepsilon_{1}$.

Table 1: $\quad$ Flat fire vertical $(\Delta y)$ and horizontal $(\Delta z)$ Coriolis deflections, calculated numerically and analytically. Projectile Lapua Scenar $.30 \mathrm{~GB} 432,185$ Grain. Gun elevation angle $\varphi_{0}=1^{\circ}$. Coriolis-free range $\mathrm{x}_{1}=1006.2 \mathrm{~m}$, hitpoint height $y_{1}=7.71 \mathrm{~m}$, distance $L_{1}=$ $1006.2 \mathrm{~m}$. Muzzle velocity $v_{0}=930 \mathrm{~m} / \mathrm{s}$, time of flight $\tau_{1}=1.6 \mathrm{~s}$.

\begin{tabular}{|c|c|c|c|c|c|}
\hline$\psi\left({ }^{\circ}\right)$ & 90 & 0 & 0 & 0 & 0 \\
\hline$\gamma\left({ }^{\circ}\right)$ & $0 \div 360$ & 0 & 90 & 180 & 270 \\
\hline$\Delta \mathrm{y}(\mathrm{mm})$ & 0 & 0 & +102 & 0 & -102 \\
\hline$\Delta \mathrm{z}(\mathrm{mm})$ & +102 & -1 & 0 & +1 & 0 \\
\hline
\end{tabular}


Table 2: Uphill fire Coriolis deflections, calculated numerically and analytically (deviating analytical results in brackets). Projectile Lapua of Table $1, \varphi_{0}=46^{\circ}, x_{1}=705.45 \mathrm{~m}, y_{1}=720.58 \mathrm{~m}, L_{1}=$ $1008,41 \mathrm{~m}, v_{0}=930 \mathrm{~m} / \mathrm{s}, \tau_{1}=1.6 \mathrm{~s}$.

\begin{tabular}{|c|c|c|c|c|c|}
\hline$\psi\left({ }^{\circ}\right)$ & 90 & 45 & 45 & 45 & 45 \\
\hline$\gamma\left({ }^{\circ}\right)$ & $0 \div 360$ & 0 & 90 & 180 & 270 \\
\hline$\Delta \mathrm{y}(\mathrm{mm})$ & 0 & 0 & +51 & 0 & $-52(-51)$ \\
\hline$\Delta \mathrm{z}(\mathrm{mm})$ & +72 & -1 & +51 & +103 & +51 \\
\hline
\end{tabular}

Table 3: Flat fire Coriolis deflections, calculated numerically and analytically (deviating analytical results in brackets). Projectile of Lapua geometry and mass density but threefold diameter $(\mathrm{d}=23.47$ $\mathrm{mm}, M=323.68 \mathrm{~g}), \varphi_{0}=2^{\circ}, x_{1}=3019.22 \mathrm{~m}, y_{1}=16.75 \mathrm{~m}, L_{1}=$ $3019.26 \mathrm{~m}, v_{0}=930 \mathrm{~m} / \mathrm{s}, \tau_{1}=4.8 \mathrm{~s}$.

\begin{tabular}{|c|c|c|c|c|c|}
\hline$\psi\left({ }^{\circ}\right)$ & 90 & 0 & 0 & 0 & 0 \\
\hline$\gamma\left({ }^{\circ}\right)$ & $0 \div 360$ & 0 & 90 & 180 & 270 \\
\hline$\Delta \mathrm{y}(\mathrm{mm})$ & 0 & 0 & +920 & 0 & -920 \\
\hline$\Delta \mathrm{z}(\mathrm{mm})$ & +920 & $-14(-17)$ & 0 & $+14(+17)$ & 0 \\
\hline
\end{tabular}

Table 4: Uphill fire Coriolis deflections, calculated numerically and analytically (deviating analytical results in brackets). Projectile of Table 3, $\varphi_{0}=47^{\circ}, x_{1}=2116.19 \mathrm{~m}, y_{1}=2178.37 \mathrm{~m}, L_{1}=3037.03 \mathrm{~m}$, $v_{0}=930 \mathrm{~m} / \mathrm{s}, \tau_{1}=4.8 \mathrm{~s}$.

\begin{tabular}{|c|c|c|c|c|c|}
\hline$\psi\left({ }^{\circ}\right)$ & 90 & 45 & 45 & 45 & 45 \\
\hline$\gamma\left({ }^{\circ}\right)$ & $0 \div 360$ & 0 & 90 & 180 & 270 \\
\hline$\Delta \mathrm{y}(\mathrm{mm})$ & 0 & 0 & $+469(+463)$ & 0 & $-469(-463)$ \\
\hline$\Delta \mathrm{z}(\mathrm{mm})$ & +655 & $-20(-22)$ & +463 & $+947(+948)$ & +463 \\
\hline
\end{tabular}

\subsection{Example 4}

The same data are used as in example 3, however the elevation angle changes to $\varphi_{0}=47^{\circ}$ and the latitude to $\psi=45^{\circ}$. The Coriolis-free distance is now $L_{1}=$ $3037.03 \mathrm{~m}$, which is slightly longer than in example 3 , although gravity pulls the projectile back. The reason is the decreasing air pressure along the trajectory. Table 4 again shows very good agreement. The largest error occurs in height with $6 \mathrm{~mm}$ at a distance of about $3 \mathrm{~km}$. As mentioned with eq. (26), in the analytical approach the corrected exponent $m$ has to be used. In this particular case $m=0.4855, n=0.3574$ (eq. (27)) and the corrected exponent $m^{*}=0.1281$.

\section{Conclusions}

The developed analytical approach yields sufficiently accurate vertical and horizontal Coriolis deflections and represents a simple and useful extension of 
the previously developed [4] analytical fast calculation method for direct fire applications.

\section{References}

[1] McCoy, R.L., Modern Exterior Ballistics / The Launch and Flight Dynamics of Symmetric Projectiles, Schiffer Publishing Ltd: Atglen, PA 19310, 1999.

[2] Roetzel, W., Analytische Berechnung gestreckter Geschossflugbahnen. Mitteilungen der Schießsport-Arbeitsgemeinschaft an der Universität der Bundeswehr Hamburg Nr. 1, Ed. H. Rothe, Helmut-Schmidt-Universität, Universität der Bundeswehr Hamburg, 2004.

[3] Kuhrt, A., Rothe H., The use of computer algebra and nonlinear optimization for realtime computation of fire orders for direct fire, Computational Ballistics II, pp. 337 -346, Eds. V. Sanchez-Galvez, C.A. Brebbia, A.A. Motta, C.E. Anderson, WIT Press 2005.

[4] Roetzel, W., Analytical calculation of trajectories using a power law for the drag coefficient variation with Mach number. Computational Ballistics II, pp. 303 - 312, Eds. V. Sanches-Golvez, C.A. Brebbia, A.A. Motta, C.E. Anderson, WIT Press 2005.

[5] Roetzel, W., Verfahren zur nachträglichen Berücksichtigung von CoriolisEffekten bei der Bestimmung der Flugbahnen von Geschossen, unpublished report, Hamburg, 2005.

[6] Rogers, G.F.C., Mayhew, Y.R., Thermodynamic and Transport Properties of Fluids, Basil Blackwell Ltd. Oxford, $4^{\text {th }}$ Edition, 1988.

[7] Szabó, I., Einführung in die Technische Mechanik, 3. Auflage, SpringerVerlag, Berlin / Göttingen / Heidelberg, 1958. 